\section{The Vancouver bobsled: cool patellofemoral runnings}

\author{
Mario Bizzini
}

In September 2013, Vancouver hosted the International Patellofemoral Pain (PFP) Research Symposium, where the best experts presented and debated the latest developments in this area. In the same city the unforgettable 2010 Winter Olympics took place, which included the bobsled competitions in Whistler. The link between these two events is that patellar tracking on the femoral trochlea is often compared with a bobsled within the ice track. The traditional view is that the patella/bobsled is unstable within the trochlea/track. However, the research at the symposium demonstrated that the patella is influenced by the position of the femur and 'follows' its orientation. To illustrate this, dynamic valgus and femoral internal rotation at the knee pushes the patella laterally, stressing the medial structures and potentially leading to PFP. These biomechanical changes in turn can affect many soft tissues (capsule, ligaments, tendons, muscles and fascia) that may be the source of the pain.

\section{PFP UPDATE}

We present the latest research from the 2013 Vancouver Symposium, the pearl being the consensus paper (see page 411). The systematic review and meta-analysis by Barton et al (see page 417) examines the role of patellar taping for PFP, in terms of biomechanical mechanisms and clinical outcomes.

The effect of surgery on the patellofemoral joint (PFJ) and the effect of

Correspondence to Mario Bizzini, Research Department, FIFA-Medical Assessment \& Research Centre, Swiss Sports Physiotherapy Association, Schulthess Clinic, Lengghalde 2, Zürich 8008, Switzerland; mario.bizzini@f-marc.com footwear on the PFJ forces are also investigated in this issue. The long-term follow-up study by Culvenor et al (see page 435) showed a high prevalence of PFJ osteoarthritis after anterior cruciate ligament reconstruction (ACLR) with hamstring autograft, something that usually is discussed only after bonepatellar-tendon-bone autograft. Because impaired function was associated with worse outcome after ACLR, the authors suggest that the PFJ should be considered in the postoperative rehabilitation.

Bonacci et al (see page 425) found that barefoot running reduced PFJ stress, and therefore this may be an interesting treatment option for PFP patients. Hedayatpour et al (see page 429) found that eccentric quadriceps exercise can impact the vastii muscle activity, and consequently may compromise patellar stabilisation. These findings may help in understanding and preventing exercise-related PFJ disorders.

The management of PFP patients still remains as challenging as driving a bobsleigh down a narrow ice track at speeds of up to 80 or $100 \mathrm{mph}$... but there is no doubt that BJSM readers will find relevant evidence-based knowledge which can be implemented in the treatment of individuals presenting with PFP, one of the most common conditions in sports medicine and sports physiotherapy clinics.

Further, there are three original papers on the Victorian Institute of Sports Assessment (VISA) score, an important patient-based measurement tool for tendon pain and function. Attia et al (see page 469) provide evidence of a relationship between the increased presence of glycosaminoglycan and the VISA-P score in patellar tendinopathy, and Hernandez-Sanchez et al (see page 453) discuss the responsiveness of the VISA-P score in patients with patellar tendinopathy. Cacchio et al (see page 448) report on a new VISA questionnaire for patients and athletes with proximal hamstring tendinopathy (VISA-H).

\section{SWISS SPORTFISIO CONFERENCE}

Bobsled is one of the popular venues for Swiss winter sports; the first tracks werebuilt in St Moritz, Switzerland around 1910. Media attention peaks during the Olympic Games where the bobsled athletes are traditionally among the favourites for Olympic medals. Swiss sports physiotherapists are actively involved in the care of the Swiss team, and these physiotherapists meet at the annual conference of the Swiss Sports Physiotherapy Association (SSPA); around 350 sports physiotherapists gathered last year in Bern. A similar number registered in Salzburg for the biennial Austrian Sports PT conference (November 2013), thus confirming the surging interest for sports physiotherapy in our alpine countries.

The 2014 SSPA conference, in Bern as usual (16 November), will host a Swiss-Austrian-German Sport PT Symposium for the first time, and will focus on 'Structures and Sports'. Speakers include renowned international speakers, Dr Peter McNair from New Zealand and Dr Antonio Stecco from Italy. Check the SSPA and BJSM websites (and the related Twitter, Facebook, blog and more) for updates on the Bern conference.

Competing interests None.

Provenance and peer review Not commissioned; internally peer reviewed.

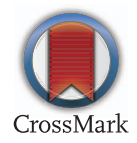

To cite Bizzini M. Br J Sports Med 2014;48:407.

Accepted 10 December 2013

Br J Sports Med 2014;48:407.

doi:10.1136/bjsports-2013-093339 